\title{
Finite volume approximation of the Anderson model
}

\author{
Fumihiko Nakano ${ }^{\text {a) }}$ \\ Faculty of Science, Department of Mathematics and Information Science, \\ Kochi University, 2-5-1 Akebonomachi, Kochi 780-8520, Japan
}

(Received 9 October 2006; accepted 23 February 2007; published online 13 April 2007)

In the Anderson model on $\mathbf{Z}^{d}$, we consider a sequence of its finite volume approximation $\left\{H_{k}\right\}_{k}$ and construct a set of sequences composed of the eigenvalues and eigenfunctions of $\left\{H_{k}\right\}$ in the localized region $I$ which converge to those of $H$ simultaneously. For its proof, Minami's estimate turns out to be important. This result implies that, in the localized region, each eigenfunction behaves almost independently around their centers of localization. (C) 2007 American Institute of Physics. [DOI: 10.1063/1.2716970]

\section{INTRODUCTION}

The model we consider in this paper is the so-called Anderson model given by

$$
(H \varphi)(x)=\sum_{|x-y|=1} \varphi(y)+V_{\omega}(x) \varphi(x),
$$

where $\left\{V_{\omega}(x)\right\}_{x \in \mathbf{Z}^{d}}$ are independent, identically distributed random variables on some probability space $(\Omega, \mathcal{F}, \mathbf{P})$ whose common distribution has the bounded density $\rho$. It is well known that the spectrum of $H$ is almost surely equal to the fixed set $\Sigma$ (Ref. 6)

$$
\sigma(H)=\Sigma:=[-2 d, 2 d]+\operatorname{supp} \rho, \quad \text { a.s. }
$$

and we can find some intervals $I(\subset \Sigma)$ such that the spectrum of $H$ on $I$ consists of densely distributed eigenvalues with exponentially decaying eigenfunctions (Anderson localization, Refs. 1,2). In this paper, we consider the sequence of boxes $\Lambda_{1} \subset \Lambda_{2} \subset \cdots$ and the restrictions $H_{k}$ := $\left.H\right|_{\Lambda_{k}}$ with suitable boundary condition to approximate $H$. We show in Theorem 2.1 that the sequences suitably chosen from the set of eigenvalues in $I$ and corresponding eigenfunctions of $\left\{H_{k}\right\}_{k}$ converge to those of $H$ and vice versa. The motivation of this problem is to examine an intuition that the locations of eigenvalues of $H$ in $I$ are determined so that the eigenvalues of all different scales are compatible.

Remark 1: Since $H_{k}$ converges to $H$ strongly, for any $\lambda \in \sigma(H)$ we have a sequence $\lambda_{k}$ $\in \sigma\left(H_{k}\right)$, with $\lambda_{k} \rightarrow \lambda$. Nevertheless, the results in Theorem 2.1 are not quite trivial as it sounds, because (i) it can happen that $\lambda_{n} \rightarrow I \backslash$ \eigenvalues of $\left.H\right\}$ and (ii) we would like to construct the set of sequences of eigenfunctions as well as eigenvalues simultaneously.

We first fix notations.

Notation

(1) For $L>0, x \in \mathbf{Z}^{d}$, let

$$
\Lambda_{L}(x):=\left\{y=\left(y_{1}, y_{2}, \ldots, y_{d}\right) \in \mathbf{Z}^{d}:\left|x_{j}-y_{j}\right| \leqslant \frac{L}{2}, \quad j=1,2, \ldots, d\right\}
$$

be the box in $\mathbf{Z}^{d}$ centered at $x$ with size $L$, and for a box $\Lambda$ let

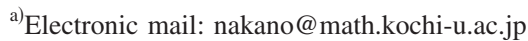




$$
\begin{gathered}
\widetilde{\partial} \Lambda:=\left\{\langle x, y\rangle \in \Lambda \times \Lambda^{c}:|x-y|=1\right\}, \\
\partial \Lambda:=\left\{x \in \Lambda:\langle x, y\rangle \in \widetilde{\partial} \Lambda \quad \text { for some } y \in \Lambda^{c}\right\}
\end{gathered}
$$

be two notions of its boundary.

(2) For a box $\Lambda$, let $H_{\Lambda}:=\left.H\right|_{\Lambda}$ be the restriction of $H$ onto $\Lambda$. We impose the Dirichlet boundary condition unless stated otherwise.

(3) Let $\gamma>0, E \in \mathbf{R}$. A box $\Lambda_{L}(x)$ is said to be $(\gamma, E)$-regular if and only if (iff) $E \notin \sigma\left(H_{\Lambda_{L}(x)}\right)$ and the following estimate holds.

$$
\left|G_{\Lambda_{L}(x)}(E ; x, y)\right| \leqslant e^{-\gamma(L / 2)}, \quad \forall y \in \partial \Lambda_{L}(x),
$$

where $G_{\Lambda}(E ; x, y):=\left\langle x\left|\left(H_{\Lambda}-E\right)^{-1}\right| y\right\rangle$ is the matrix element of Green's function.

(4) For $E \in \mathbf{R}, \delta>0, I(E, \delta):=(E-\delta, E+\delta)$ is an open interval with center $E$ and width $2 \delta$.

(5) For $\phi \in l^{2}\left(\mathbf{Z}^{d}\right)$, let

$$
X(\phi):=\left\{x \in \mathbf{Z}^{d}:|\phi(x)|=\max _{y \in \mathbf{Z}^{d}}|\phi(y)|\right\}
$$

be the set of centers of localization which is defined by Ref. 3 by which they studied the dynamical localization property of $H$. To be free from ambiguities, we choose a unique point $x(\phi) \in X(\phi)$ according to certain order on $\mathbf{Z}^{d}$. This procedure will cause no essential problems in our situation, for the elements of $X(\phi)$ are not far apart from each other in Anderson localization. For an eigenvalue $E$ of $H$, we pick the corresponding eigenfunction $\phi$ and let $X(E)=X(\phi), x(E)=x(\phi)$. In the case of degeneracy, we fix eigenfunctions along some procedure. For a box $C \subset \Lambda$, we say $\phi$ (respectively $E$ ) is localized in $C$ iff $x(\phi) \in C$ [respectively $x(E) \in C]$.

(6) For the Hamiltonian $H=H_{\Lambda}$, interval $J(\subset \mathbf{R})$, and a box $C(\subset \Lambda)$, we define

$$
\begin{gathered}
\mathcal{E}(H, J):=\{\text { eigenvalues of } H \text { in } J\}, \\
\mathcal{E}(H, J, C):=\{\text { eigenvalues of } H \text { in } J \text { localized in } C\}, \\
\mathcal{E} f(H, J):=\{\text { normalized eigenfunctions of } H \text { in } J\}, \\
\mathcal{E} f(H, J, C):=\{\text { normalized eigenfunctions of } H \text { in } J \text { localized in } C\}, \\
N(H, J):=\# \mathcal{E}(H, J), \\
N(H, J, C):=\# \mathcal{E}(H, J, C) .
\end{gathered}
$$

We consider the following assumption for an interval $I=(a, b)(\subset \Sigma)$.

\section{Assumption}

We can find $p(>4 d), \gamma>0$ such that

$$
\mathbf{P}\left(\text { For any } E \in I, H_{\Lambda_{L_{0}}(0)} \text { is }(\gamma, E) \text {-regular }\right) \geqslant 1-L_{0}^{-p}
$$

for large $L_{0}$.

Assumption is known to hold for some regions (band edges, extreme energies, etc.) in $\Sigma$, and by which we can deduce that Anderson localization holds on $I$ via the multiscale analysis. ${ }^{10}$ Take $\alpha$ with $1<\alpha<2 p /(p+2 d)$ and let $L_{k+1}:=L_{k}^{\alpha}, k=0,1,2, \ldots$. Then by Ref. 10, we have 
$\mathbf{P}\left(\right.$ For $\forall E \in I$, either $\Lambda_{k}(x)$ or $\Lambda_{k}(y)$ is $(\gamma, E)$-regular $) \geqslant 1-L_{k}^{-2 p}$,

for $k=1,2, \ldots$ and for any $x, y \in \mathbf{Z}^{d}$ with $|x-y|>L_{k}$.

\section{CONVERGENCE OF EIGENVALUES AND EIGENFUNCTIONS}

Let $\Lambda_{k}:=\Lambda_{L_{k}}(0)$ be the box with size $L_{k}$ centered at the origin and let $H_{k}:=\left.H\right|_{\Lambda_{k}}$ with periodic boundary condition. Let

$$
\begin{gathered}
C_{k}:=\left\{x \in \Lambda_{k}: d\left(x, \partial \Lambda_{k}\right) \geqslant L_{k-1}\right\}, \\
D_{k}:=\left\{x \in \Lambda_{k}: d\left(x, \partial \Lambda_{k}\right) \geqslant 2 L_{k-1}\right\}, \\
F_{k}:=\left\{x \in \Lambda_{k}: d\left(x, \partial \Lambda_{k}\right) \geqslant 4 L_{k-1}\right\}
\end{gathered}
$$

be boxes in $\Lambda_{k}$. We further take any $0<\gamma^{\prime}<\gamma$ and let

$$
\begin{gathered}
\epsilon(k):=\sum_{l \geqslant k} \epsilon_{l-1}, \quad \epsilon_{l}:=\mathrm{e}^{-\gamma^{\prime} L_{l} / 2}, \\
I=(a, b), \quad I_{k}:=(a+\epsilon(k), b-\epsilon(k)) .
\end{gathered}
$$

Theorem 2.1: For a.e. $\omega$, we can find $K(\omega)=K\left(\omega, \alpha, d, \gamma, \gamma^{\prime}\right)$ such that the following facts hold for $k \geqslant K(\omega)$ : we can construct the one to one map

$$
\varphi_{k, k+1}: \mathcal{E}\left(H_{k}, I_{k}, D_{k}\right) \rightarrow \mathcal{E}\left(H_{k+1}, I_{k+1}, \Lambda_{k}\right),
$$

with the following property. Let

$$
\begin{gathered}
\{E(j ; K(\omega), K(\omega))\}_{j}=\mathcal{E}\left(H_{K(\omega)}, I_{K(\omega)}, D_{K(\omega)}\right), \\
\{E(j ; k, k)\}_{j}=\mathcal{E}\left(H_{k}, I_{k}, D_{k}\right) \backslash \varphi_{k-1, k}\left(\mathcal{E}\left(H_{k-1}, I_{k-1}, D_{k-1}\right)\right), \quad k>K(\omega), \\
E(j ; k, m)=\varphi_{m-1, m} \circ \varphi_{m-2, m-1} \circ \cdots \circ \varphi_{k, k+1}(E(j ; k, k)), \quad m>k,
\end{gathered}
$$

then the limit

$$
E(j, k):=\lim _{m \rightarrow \infty} E(j ; k, m)
$$

exists for any $j, k$ and are eigenvalues of $H$ on $I$. Furthermore, the corresponding normalized eigenfunctions $\phi_{j, k, m}$ of $H_{m}$ converge to those of $H$ in $l^{2}\left(\mathbf{Z}^{d}\right)$, and $X(E(j, k)) \subset \Lambda_{k}$.

Conversely, for any eigenvalue $E$ of $H$ on $I_{K(\omega)}$, we can uniquely find the sequence $\{E(j ; k, m)\}_{m}$ converging to $E$.

Proof: We first consider the following event.

$$
\begin{aligned}
\Omega_{k}:= & \left\{\omega \in \Omega \text { : For any } E \in I, \text { either } \Lambda_{k-1}(x) \text { of } \Lambda_{k-1}(y) \text { is }(\gamma, E)\right. \text {-regular } \\
& \text { for any } \left.x, y \in \Lambda_{k+1} \text { with }|x-y|>L_{k-1}\right\} .
\end{aligned}
$$

Since we have $\mathbf{P}\left(\Omega_{k}^{c}\right) \leqslant($ const $)\left|\Lambda_{k+1}\right|^{2} L_{k-1}^{-2 p}=$ (const) $L_{k-1}^{2 \alpha^{2} d-2 p}$ by Eq. (1.2) and since $p>4 d, \Omega_{0}$ $:=\lim _{\inf _{k \rightarrow \infty}} \Omega_{k}$ satisfies $\mathbf{P}\left(\Omega_{0}\right)=1$. For $\omega \in \Omega_{0}$, we can find $k_{0}(\omega)$ such that $\omega \in \Omega_{k}$ whenever $k$ $\geqslant k_{0}(\omega)$. We next use the argument in Ref. 5 here: let 


$$
d_{k}:=\frac{1}{\left|\Lambda_{k}\right|^{2} k^{2}}
$$

and divide $I=\cup_{j=1}^{N_{k}}\left[x_{j}, x_{j+1}\right],\left|x_{j}-x_{j+1}\right|=d_{k}, N_{k}=([|I|]+1) / d_{k}$. Let $I_{j}:=I\left(x_{j}, d_{k}\right)$ and cover $I$ by overlapping intervals $\left\{I_{j}\right\}_{j}$. We consider the following events.

$$
\widetilde{\Omega}_{k}:=\left\{\omega \in \Omega: N\left(H_{k}, I_{j}\right) \leqslant 1 \text { for any } j=1,2, \ldots, N_{k}\right\} .
$$

For $\omega \in \widetilde{\Omega}_{k}$, the minimum spacing of the eigenvalues of $H_{k}$ is larger than $d_{k}$. By Minami's estimate, ${ }^{7}$ we have

$$
\mathbf{P}\left(\widetilde{\Omega}_{k}^{c}\right) \leqslant \pi^{2}\|\rho\|_{\infty}^{2} \sum_{j=1}^{N_{k}}\left|I_{j}\right|^{2}\left|\Lambda_{k}\right|^{2}=\text { (const) } \frac{1}{d_{k}} d_{k}^{2}\left|\Lambda_{k}\right|^{2}=\frac{\text { (const) }}{k^{2}}
$$

so that $\widetilde{\Omega}_{0}:=\lim \inf _{k \rightarrow \infty} \widetilde{\Omega}_{k}$ satisfies $\mathbf{P}\left(\widetilde{\Omega}_{0}\right)=1$, and hence for $\omega \in \widetilde{\Omega}_{0}$ we can find $\widetilde{k}_{0}(\omega)$ such that $\omega \in \widetilde{\Omega}_{k}$ for $k \geqslant \widetilde{k}_{0}(\omega)$. Let $\omega \in \Omega_{0} \cap \widetilde{\Omega}_{0}$ and take $K(\omega) \geqslant k_{0}(\omega) \vee \widetilde{k}_{0}(\omega) \vee k_{3}\left(\alpha, d, \gamma, \gamma^{\prime}\right)$ large enough with $2 \epsilon_{K-1}<d_{K+1}{ }^{1}$. Then by Lemma 3 , if $k \geqslant K(\omega)$, for any $E \in \mathcal{E}\left(H_{k}, I_{k}, D_{k}\right)$, there is unique element $\varphi_{k, k+1}(E) \in I\left(E, \epsilon_{k-1}\right) \cap \mathcal{E}\left(H_{k+1}, I_{k+1}, \Lambda_{k}\right)$ which is the definition of the map $\varphi_{k, k+1}: \mathcal{E}\left(H_{k}, I_{k}, D_{k}\right) \rightarrow \mathcal{E}\left(H_{k+1}, I_{k+1}, \Lambda_{k}\right)$. Noting $\mathcal{E}\left(H_{k+1}, I_{k+1}, \Lambda_{k}\right) \subset \mathcal{E}\left(H_{k+1}, I_{k+1}, D_{k+1}\right)$ for large $k$, we can iterate this procedure. On the other hand, elements in $\mathcal{E}\left(H_{k+1}, I_{k+1}, \Lambda_{k}\right) \backslash \varphi_{k, k+1}\left(\mathcal{E}\left(H_{k}, I_{k}, D_{k}\right)\right)$ and in $\mathcal{E}\left(H_{k+1}, I_{k+1}, D_{k+1} \backslash \Lambda_{k}\right)$ are the ones which first appear in the $(k+1)$ th step, which we denote

$$
\{E(j ; k+1, k+1)\}_{j=1}^{N_{k+1}}:=\mathcal{E}\left(H_{k+1}, I_{k+1}, D_{k+1}\right) \backslash \varphi_{k, k+1}\left(\mathcal{E}\left(H_{k}, I_{k}, D_{k}\right)\right) .
$$

Then the set of all eigenvalues we have up to the $k$ th step is given by $\cup_{l=K(\omega)}^{k}\{E(j ; l, k)\}_{j=1}^{N_{l}}$, where we set

$$
E(j ; l, k):=\varphi_{k-1, k} \circ \varphi_{k-2, k-1} \circ \cdots \circ \varphi_{l, l+1}(E(j ; l, l))
$$

for $k>l$. Since $\left|E-\varphi_{k, k+1}(E)\right| \leqslant \epsilon_{k-1}$ for $E \in \mathcal{E}\left(H_{k}, I_{k}, D_{k}\right)$,

$$
E(j ; l)=\lim _{k \rightarrow \infty} E(j ; l, k)
$$

exists. To show that they are the eigenvalues of $H$, we have to study the correspondence between eigenfunctions.

Claim 1: For any $k \geqslant k_{3}\left(\alpha, d, \gamma, \gamma^{\prime}\right)$, we can find $\rho=\rho\left(\alpha, d, \gamma, \gamma^{\prime}\right)>0$ with the following properties. If $\omega \in \Omega_{k} \cap \widetilde{\Omega}_{k}$ and if $\phi \in \mathcal{E} f\left(H_{k},\{E\}, D_{k}\right), E \in I_{k}$ and $\widetilde{\phi} \in \mathcal{E} f\left(H_{k+1},\left\{\varphi_{k, k+1}(E)\right\}, \Lambda_{k}\right)$, we have

$$
\|\phi-\widetilde{\phi}\|_{l^{2}\left(\Lambda_{k+1}\right)} \leqslant \mathrm{e}^{-\rho L_{k-1}} .
$$

Proof of Claim 1: Let $P$ be the spectral projection of $H_{k+1}$ corresponding to $I\left(E, \epsilon_{k-1}\right)$. Then we have $\widetilde{\phi}=P \phi /\|P \phi\|_{l^{2}\left(\Lambda_{k+1}\right)}$ and by the argument of the proof of Lemma 3, $\|\phi-P \phi\|_{l^{2}\left(\Lambda_{k+1}\right)}$ $\leqslant(1 / 2) \mathrm{e}^{-\rho L_{k-1}}$ for some $\rho>0$.

By Claim $1, \phi_{j, l, k} \in \mathcal{E} f\left(H_{k},\{E(j ; l, k)\}, D_{k}\right)$ converges to some $\phi_{j, l}$ in $l^{2}\left(\mathbf{Z}^{d}\right)$ which implies $E(j, l)$ is an eigenvalue of $H$ with $\phi_{j, l}$ corresponding to normalized eigenfunctions. $X(E(j, l)) \subset \Lambda_{l}$ follows from the following claim.

Claim 2: We can find $k_{0}=k_{0}(\alpha, d, \rho)$ with the following properties. If $k \geqslant k_{0}$, and if $\varphi$ $\in l^{2}\left(\Lambda_{k}\right), \phi \in l^{2}\left(\mathbf{Z}^{d}\right)$ satisfy $\|\varphi\|_{l^{2}\left(\Lambda_{k}\right)}=\|\phi\|_{l^{2}\left(\mathbf{Z}^{d}\right)}=1, x(\varphi) \in D_{k}$, and $\|\varphi-\phi\|_{l^{2}\left(\mathbf{Z}^{d}\right)} \leqslant \mathrm{e}^{-\rho L_{k-1}}$, then $x(\phi)$ $\in \Lambda_{k}$.

Proof of Claim 2: Suppose $x(\phi) \in \Lambda_{k}^{c}$. Then $|\phi(x)| \leqslant \mathrm{e}^{-\rho L_{k-1}}$ for $x \in \Lambda_{k}$ and $\|\phi\|_{l^{2}\left(\Lambda_{k}^{c}\right)}^{2} \leqslant \mathrm{e}^{-2 \rho L_{k-1}}$.

${ }^{1} k_{3}\left(\alpha, d, \gamma, \gamma^{\prime}\right), k_{4}\left(\alpha, d, \gamma, \gamma^{\prime}\right)$ are constants given in Lemmas 3 and 4 in the Appendix depending only on $\alpha, d, \gamma, \gamma^{\prime}$. 
Taking $k_{0}(\alpha, d, \rho)$ large enough with $\left(\left|\Lambda_{k}\right|+1\right) \mathrm{e}^{-2 \rho L_{k-1}<1}$ leads us to a contradiction.

To show the converse statement, we pick $E \in \mathcal{E}\left(H, I_{K(\omega)}\right)$ and take $k$ large enough with $x(E)$ $\in F_{k}$. By Lemma 4, if $k \geqslant k_{0}(\omega) \vee k_{4}\left(\alpha, d, \gamma, \gamma^{\prime}\right) \vee K(\omega)$ is large enough, we can uniquely find $l$ $\geqslant K(\omega)$ with $E(j ; l, k) \in \mathcal{E}\left(H_{k}, I_{k}, D_{k}\right) \cap I\left(E, \epsilon_{k-1}\right)$. The proof of Theorem 1 is completed.

Remark 1: Since $\phi_{j, l, k} \perp \phi_{j^{\prime}, l^{\prime}, k}$ for $(j, l) \neq\left(j^{\prime}, l^{\prime}\right)$ and since the eigenvalues of $H$ are simple, ${ }^{5}$ $E(j, l) \neq E\left(j^{\prime}, l^{\prime}\right)$ for $(j, l) \neq\left(j^{\prime}, l^{\prime}\right)$. Thus $\{E(j, l)\}$ is a labeling of eigenvalues of $H$.

Remark 2: We can easily construct sequences which are composed of $\{E(j ; l, k)\}_{l, k, j}$ and converge to the elements of $I \backslash$ \{eigenvalues of $H$ \}. In fact, for any $F \in I \backslash$ \{eigenvalues of $H$ \}, we can find an eigenvalue $E\left(j_{k}, l_{k}\right)$ of $H$ with $\left|F-E\left(j_{k}, l_{k}\right)\right|<1 / k$ for any $k$. Since $\lim _{k \rightarrow \infty} l_{k}=\infty$, $\lim _{k \rightarrow \infty} E\left(j_{k} ; l_{k}, l_{k}\right)=F$.

Remark 3: The proof of Theorem 1 suggests that the finite volume eigenfunctions do not move too much to become the infinite volume ones modulo exponentially small error. It also suggests that to construct the set of sequences of finite box eigenvalues which converge to the dense point spectrum simultaneously, one needs extra information (such as Minami's estimate) to construct the correspondence between eigenfunctions of different boxes. In other words, Minami's estimate tells us that eigenfunctions localize around their centers in space almost independently from each other, so that the finite volume approximation $H \simeq \oplus_{k} H_{k}$ holds not only in the sense of eigenvalues but also in eigenfunctions.

This situation contrasts with that in Ref. 9 (cf. Ref. 4), where we studied the point process whose atoms are eigenvalues and their centers. There we needed the correspondence between eigenvalues only to prove the infinite divisibility of the limiting point process, so that Minami's estimate was not necessary.

\section{APPENDIX}

We collect the basic facts used in this paper, whose proofs are only sketched or omitted, for they are elementary or already given elsewhere (e.g., Refs. 3, 5, 8, and 9).

The following two lemmas give us the exponential decay of eigenfunctions away from their center of localization.

Lemma 1 (Ref. 3): Let $H \phi=E \phi, \phi \in l^{2}\left(\mathbf{Z}^{d}\right)$. Then $\Lambda_{L}(x(\phi))$ is $(\gamma, E)$-singular if $L \geqslant L_{0}$ for some $L_{0}(d, \gamma)$.

Take $k_{1}(\alpha, d, \gamma)$ with $L_{k_{1}}>L_{0}(d, \gamma)$.

Lemma 2: For any $0<\gamma_{1}<\gamma$, we can find $k_{2}\left(\alpha, d, \gamma, \gamma_{1}\right)$ with the following properties. If $k$ $\geqslant k_{2}, \omega \in \Omega_{k}, k-1 \geqslant k_{1}(\alpha, d, \gamma), H_{k} \phi=E \phi, E \in I,\|\phi\|_{l^{2}\left(\Lambda_{k}\right)}=1$, and $x(\phi) \in C_{k}$, then

$$
|\phi(y)| \leqslant e^{-\gamma_{1}\left(L_{k-1}\right) / 2}, \quad y \in \partial \Lambda_{k} .
$$

To prove Lemma 2, we use the formula

$$
\phi(x)=\sum_{\left\langle y, y^{\prime}\right\rangle \in \tilde{\partial} \Lambda_{k-1}(x)} G_{\Lambda_{k-1}(x)}(E ; x, y) \phi\left(y^{\prime}\right)
$$

and the fact that $\Lambda_{k-1}(x)$ is $(\gamma, E)$-regular, since $\Lambda_{k-1}(x(\phi))$ is $(\gamma, E)$-singular and $\omega \in \Omega_{k}$. This is the place where the periodic boundary condition on $H_{k}$ is used.

When Anderson localization holds, an eigenvalue of small box Hamiltonian produces one of the bigger boxes and vice versa. More precisely, the following facts (Lemmas 3 and 4) are derived by using Lemmas 1 and 2. They are essentially proven in Ref. 5, Lemma 1, and Ref. 9, Lemmas 4.7 and 4.4 but here we need the control of the centers of localization. For the rest of this section, we take any $\gamma^{\prime}$ with $0<\gamma^{\prime}<\gamma$ and any interval $J(\subset I)$.

Lemma 3: We can find $k_{3}=k_{3}\left(\alpha, d, \gamma, \gamma^{\prime}\right)$ such that if $k \geqslant k_{3}$ and $\omega \in \Omega_{k}$, then

$$
N\left(H_{k}, J, D_{k}\right) \leqslant N\left(H_{k+1}, J+I\left(0, \epsilon_{k-1}\right), \Lambda_{k}\right) .
$$

Idea of proof: Let $M:=N\left(H_{k}, J, D_{k}\right)$ and let $P$ be the spectral projection of $H_{k+1}$ corresponding 
to $J+I\left(0, \epsilon_{k-1}\right)$. Since $\phi_{1}, \ldots, \phi_{M} \in \mathcal{E} f\left(H_{k}, J, D_{k}\right)$ decay exponentially on $C_{k}^{c}$, so are $P \phi_{1}, \ldots, P \phi_{M}$. We can write

$$
P \phi_{1}=\psi_{1}+\psi_{1}^{\prime}, \ldots, P \phi_{M}=\psi_{M}+\psi_{M}^{\prime}
$$

where $\left\{\psi_{j}\right\} \subset \operatorname{Span} \mathcal{E} f\left(H_{k+1}, J+I\left(0, \epsilon_{k-1}\right), \Lambda_{k}\right),\left\{\psi_{j}^{\prime}\right\} \subset \operatorname{Span} \mathcal{E} f\left(H_{k+1}, J+I\left(0, \epsilon_{k-1}\right), \Lambda_{k}^{c}\right)$. Since $\left\{P \phi_{l}\right\}$ are ONS on $l^{2}\left(C_{k}\right)$ modulo the exponential error, and since $\psi_{j}^{\prime}$ decays exponentially on $C_{k}$, $\psi_{1}, \ldots, \psi_{M}$ are linearly independent so that $N\left(H_{k+1}, J+I\left(0, \epsilon_{k-1}\right), \Lambda_{k}\right) \geqslant M$.

We do the converse. Let

$$
\Omega_{\mathrm{loc}}:=\{\omega \in \Omega: \text { generalized eigenfunction on } I \text { decays exponentially }\}
$$

which satisfies $\mathbf{P}\left(\Omega_{\text {loc }}\right)=1$ by Ref. 10 .

Lemma 4: We can find $k_{4}=k_{4}\left(\alpha, d, \gamma, \gamma^{\prime}\right)$ such that, if $k \geqslant k_{4}$ and $\omega \in \cap_{l \geqslant k} \Omega_{l} \cap \Omega_{\text {loc }}$, we have

$$
N\left(H, J, F_{k}\right) \leqslant N\left(H_{k}, J+I\left(0, \epsilon_{k-1}\right), D_{k}\right) .
$$

Lemma 4 is proven similarly as Lemma 3.

\section{ACKNOWLEDGMENT}

This work is partially supported by JSPS grant KibanC No. 18540125.

${ }^{1}$ Aizenman, M. and Molchanov, S., "Localization at large disorder and at extreme energies: An elementary derivation," Commun. Math. Phys. 157, 245-278 (1993).

${ }^{2}$ Fröhlich, J. and Spencer, T., "Absence of diffusion in the Anderson tight binding model for large disorder or low energy," Commun. Math. Phys. 88, 151-184 (1983).

${ }^{3}$ Germinet, F. and De Bièvre, S., "Dynamical localization for discrete and continuous random Schrödinger operators," Commun. Math. Phys. 194, 323-341 (1998).

${ }^{4}$ Killip, R. and Nakano, F., "Eigenfunction statistics in the localized Anderson model," Ann. Henri Poincare 8(1), 27-36 (2007).

${ }^{5}$ Klein, A. and Molchanov, S., "Simplicity of eigenvalues in the Anderson model," J. Stat. Phys. 122, 95-99 (2006).

${ }^{6}$ Kunz, H. and Souillard, B., "Sur le spectre des operateurs aux differences finies aleatoires," Commun. Math. Phys. 78, 201-246 (1980).

${ }^{7}$ Minami, N., "Local fluctuation of the spectrum of a multidimensional Anderson tight binding model," Commun. Math. Phys. 177, 709-725 (1996).

${ }^{8}$ Nakano, F., "Repulsion between localization centers in the Anderson model," J. Stat. Phys. 123, 803-810 (2006).

${ }^{9}$ Nakano, F., "The distribution of localization centers in some discrete random systems," math-ph/0701042.

${ }^{10}$ von Dreifus, H. and Klein, A., "A new proof of localization in the Anderson tight binding model," Commun. Math. Phys. 124, 285-299 (1989). 
Journal of Mathematical Physics is copyrighted by the American Institute of Physics (AIP). Redistribution of journal material is subject to the AIP online journal license and/or AIP copyright. For more information, see http://ojps.aip.org/jmp/jmpcr.jsp 